\title{
Linear Free Vibration Analysis of Tapered Rectangular Cantilevered Timoshenko Beams using Energy Field Method
}

\author{
Shaik Mahmad raffi ${ }^{1 *}$, Ch. Tirumala Rao ${ }^{2}$ \\ ${ }^{1}$ M. Tech Scholar, Acharya Nagarjuna University, Guntur, Andhra Pradesh, India \\ ${ }^{2}$ Assistant Professor, Acharya Nagarjuna University, Guntur, Andhra Pradesh, India \\ *Corresponding author E-mail:Shaikrafi081@gmail.com
}

\begin{abstract}
Here in this paper we discuss about Energy field method. There are many methods to evaluate the natural frequency of the structures but in this research paper the authors developed a method called "Energy field method" which reduces computational efforts compared with the other methods and which is successfully applied for the Cantilever boundary condition of a tapered (rectangular cross section) Timoshenko beam and calculated the fundamental frequency parameter values and compared the results with existing literature. To confirm the precision, coherence and adaptability of the model these resultant values are also compared with modal structural analysis values in Ansys 10 software.
\end{abstract}

Keywords: linear free vibrations, Energy field method, tapered Timoshenko beams, taper ratio, and slenderness ratio.

\section{Introduction}

The cantilever beam is a constructional piece in which one end is fixed supported and other end is free. The connection of beam with support is usually perpendicular and beam is vertical. For a normal cantilever beam we can consider only shear modulus but in case if Timoshenko cantilever beam we can consider both normal and rotational effects at a time.

Vibrations are the mechanical structures experience whereby swinging backwards and forwards occurs about the equipoise spot. When a mechanical structures is in swinging by its own weight that vibrations are called free vibrations. The frequencies of those vibrations are called as natural frequencies of the structure. Vibration motion of a mechanical structure could understand by conservation of energy.

In the present method the concept of Energy Field Method is presented for the vibration analysis of tapered Timoshenko cantilevered beams, where the transverse displacement is expressed in terms of total rotation and its derivatives depending on the transverse shear deformation theory, in which the number of un known coefficients is brought down

to exactly hk/l in the coupled displacement field method. To demonstrate the efficiency of the proposed method, the expressions for fundamental frequency are obtained for tapered shear flexible Timoshenko beams with Cantilever type of boundary condition. The numerical results presented in this paper are matching very closely with the existing literature.

ANSYS is a mechanical simulation and analysis software. ANSYS mechanical software offers an inclusive product solution for systematic linear, non-linear and dynamics analysis. This simulation software offers a complete set of unit action, material models and equation solvers for a baggy variety of engineering problems. The ANSYS mechanical software is entrust by federations on all sides of the whole world to expeditiously find the key to multiplex structural and thermal problems with effect less. Using ANSYS Mechanical software exposition, you can carry out analyses to judge the strength, vibration, motion and thermal response attributes of the apparatus. An assortment of graphical contraption grants you to nimbly think about the results of your simulation.

\section{Energy field method}

\subsection{Coupling equation}

According to law conversation of energy total energy of the system at any state is constant applying the same principle we can develop the present concept. The concept of coupled displacement field is explained in detail. In the Energy Field Method with the single term admissible function for Eita, the displacement function for $\mathrm{w}$ is derived using the coupling equation.

While the beam is in stationary position the sum of strain energy and potential energy is constant $(\mathrm{U}+\mathrm{W}=$ constant $)$. When the beam is free to vibrate by its own weight the sum of strain energy and kinetic energy is constant $(\mathrm{U}+\mathrm{K} . \mathrm{E}=$ constant $)$ this is the basic principle of energy field method. For derivation of coupling and frequency function we can use the principle of minimization of potential energy from FEM equations. 


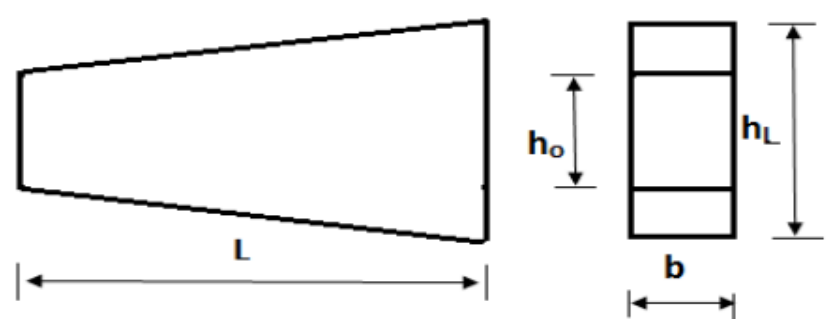

Fig. 1: Tapered Timoshenko beam with linearly varying height (depth)

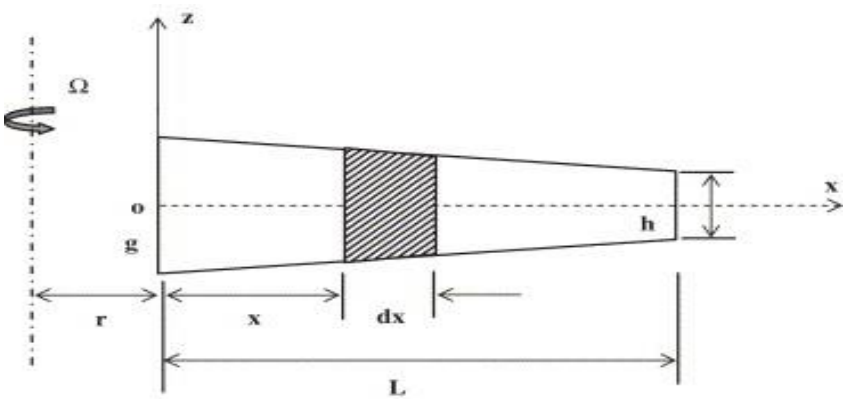

Fig.2: Tapered (rectangular cross section) Timoshenko Cantilever beam

\subsection{Free vibrations}

From The kinematics of a shear flexible beam theory (based on the Timoshenko beam theory):

Axial displacement $(\mathrm{u})=\mathrm{Z}_{\theta}$

Transverse $=\omega$

Axial strain, $t_{x}=\frac{\partial u}{\partial x}=z \frac{\partial \theta}{\partial x}$

Shear strain $\gamma_{x z}=\frac{\partial \omega}{\partial x}+\frac{\partial \omega}{\partial z}=\frac{\partial \omega}{\partial x}+\theta$

Strain energy is given by,

$U=\frac{E I}{2} \int_{0}^{L}\left(\frac{d \theta}{d x}\right)^{2} d x+\frac{K G A}{2} \int_{0}^{L}\left(\frac{\partial \omega}{\partial x}+\theta\right)^{2} d x$

Work done is,

$\mathrm{W} . \mathrm{D}=\int_{0}^{L} \mathrm{p}(x) \omega(x) d x$

Applying the principle of minimization of total potential energy,

$\delta(U-\omega)=0$

From equations 1 and 2, we get,

$K G A\left(\frac{d^{2} \omega}{d x^{2}}+\frac{d \theta}{d x}\right)+\mathrm{p}=0$

$E I\left(\frac{d^{2} \theta}{d x^{2}}\right)-K G A\left(\frac{d \omega}{d x}+\theta\right)=0$

These are governing equations

\subsection{Linear free vibrations}

Using the principle of conservation of total energy at any instant of time U+K.E = constant. As the depth of the beam is not uniform throughout the length, the expression for $U$ and K.E are given as:
Using the principle of conservation of total energy at any instant of time,

$\mathrm{U}+\mathrm{K} . \mathrm{E}=$ Constant

Strain energy,

$U=\frac{E}{2} \int_{0}^{L} I\left(\frac{d \theta}{d x}\right)^{2} d x+\frac{K G}{2} \int_{0}^{1} A\left(\frac{d \omega}{d x}+\theta\right)^{2} d x$

Kinetic energy is,

$K . E=\frac{\rho \omega^{2}}{2} \int_{0}^{L} A w d x+\frac{\rho \omega^{2}}{2} \int_{0}^{L} I \theta^{2} d x$

Where,

$I=I_{0}\left(1-\frac{d x}{L}\right)^{3} ; A=A_{0}\left(1-\frac{\partial x}{L}\right) ; \alpha=\frac{h_{L}-h_{0}}{h_{L}}$

By substituting all equations, we get

$$
U=\frac{E I a^{2} \pi^{4}}{4 L^{3}}\left(1-1.5 \alpha+\alpha^{2}-0.25 \alpha^{3}\right)+\frac{E I a^{2} \pi^{6}}{4 L^{5}}(1-0.5 \alpha)
$$

And,

$$
\begin{aligned}
K . E=\frac{A L a^{2} \rho \omega^{2}}{4}[1 & \left.+\left(\frac{\pi}{L}\right)^{2} \eta\right]^{2}(1-0.5 \alpha) \\
& +\frac{I_{0} a^{2} \pi^{2} \rho \omega^{2}}{4}\left(1-1.5 \alpha+\alpha^{2}-0.25 \alpha^{3}\right)
\end{aligned}
$$

Applying principle of minimization of total potential energy,

$\frac{\partial(U-K \cdot E)}{\partial a}=0$

$=\frac{\omega^{2}}{L^{8}\left(A \rho L\left[1+\left(\frac{\pi}{L}\right)^{2} \eta\right]^{4} L^{5}(1-0.5 \alpha)+I \rho \pi^{2}\left(1-1.5 \alpha+\alpha^{2}-0.25 \alpha^{3}\right)\right.}$

Where,

$\mathrm{E}=$ Young's Modulus of Elasticity

$\mathrm{I}=$ Mass moment of inertia

$\mathrm{K}=$ Shear correction factor $(\mathrm{K}=5 / 6)$

$\mathrm{G}=$ Shear modulus of Rigidity

$\mathrm{A}=$ Area of the beam

$\mathrm{L}=$ Length of the beam

$\mathrm{h}=$ Height of the beam

Tabular columns \& graphs BEAM I $(\mathrm{L}=100 \mathrm{~mm})$ 
Taper Ratio

0.1
0.2
0.3
0.4
0.5
0.6
0.7
0.8
0.9

Frequencies Values

Theoritcal Reference Analytical Ansys

$\begin{array}{rrrr}88.3287 & 87.6651 & 85.3804 & 89.334 \\ 87.7 & 89.037 & 88.3883 & 92.165 \\ 87.32 & 90.4341 & 89.4642 & 93.252 \\ 87.27 & 91.8642 & 90.1235 & 94.62 \\ 87.64 & 93.3416 & 93.8034 & 96.251 \\ 88.65 & 95.9175 & 95.2588 & 97.529 \\ 90.47 & 96.4613 & 98.7659 & 98.129 \\ 94.21 & 98.1965 & 105.6823 & 102.961 \\ 104.7 & 100.539 & 113.3123 & 106.195\end{array}$

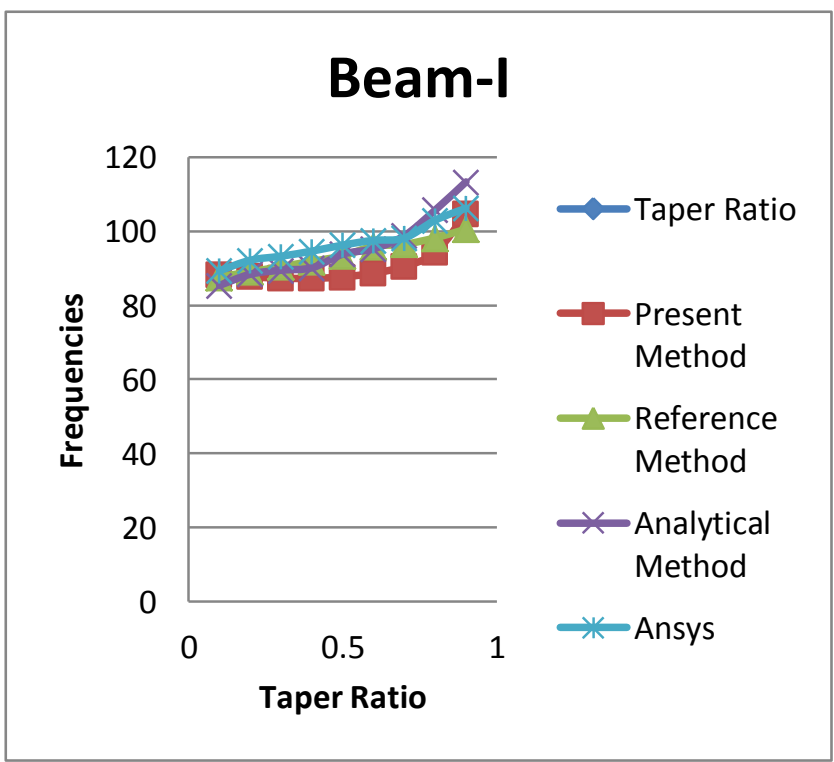

BEAM II ( $\mathbf{L}=150 \mathrm{~mm})$

Taper Ratio

Frequencies Values

Theoritcal Reference Analytical Ansys

$\begin{array}{lrrrr}0.1 & 60 & 62.429 & 59.9992 & 59.645 \\ 0.2 & 61.21 & 64.0259 & 62.5058 & 68.751 \\ 0.3 & 62.4 & 65.68 & 64.2037 & 72.08 \\ 0.4 & 63.6751 & 67.3756 & 66.4012 & 74.297 \\ 0.5 & 65.16 & 69.1769 & 67.2955 & 75.996 \\ 0.6 & 66.9872 & 71.0965 & 72.5205 & 77.429 \\ 0.7 & 69.3786 & 73.1735 & 76.5638 & 78.689 \\ 0.8 & 72.6754 & 75.4548 & 79.7526 & 79.828 \\ 0.9 & 78.8374 & 78.0961 & 86.6377 & 84.867\end{array}$
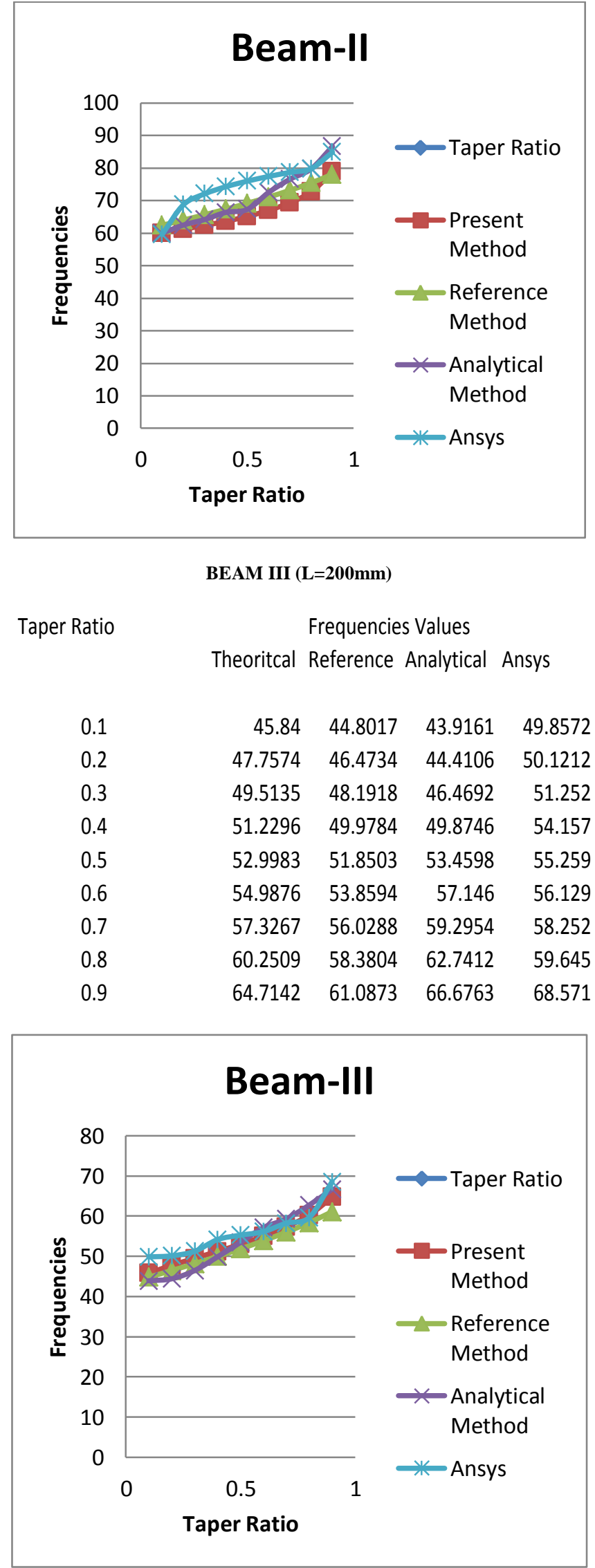

BEAM IV (L=250mm) 
Taper Ratio

Frequencies Values

Theoritcal Reference Analytical Ansys

$\begin{array}{lrrrr}0.1 & 37.28 & 40.22 & 34.1994 & 36.3097 \\ 0.2 & 39.51 & 41.9222 & 36.8042 & 38.129 \\ 0.3 & 41.4749 & 43.6509 & 39.2076 & 43.521 \\ 0.4 & 43.2966 & 45.4396 & 42.9716 & 45.527 \\ 0.5 & 45.0427 & 47.2808 & 45.718 & 48.127 \\ 0.6 & 47.0137 & 49.3165 & 49.6404 & 51.919 \\ 0.7 & 49.163 & 51.4707 & 53.3923 & 52.987 \\ 0.8 & 51.7112 & 53.8101 & 57.3572 & 55.252 \\ 0.9 & 55.195 & 56.4502 & 62.0246 & 59.765\end{array}$

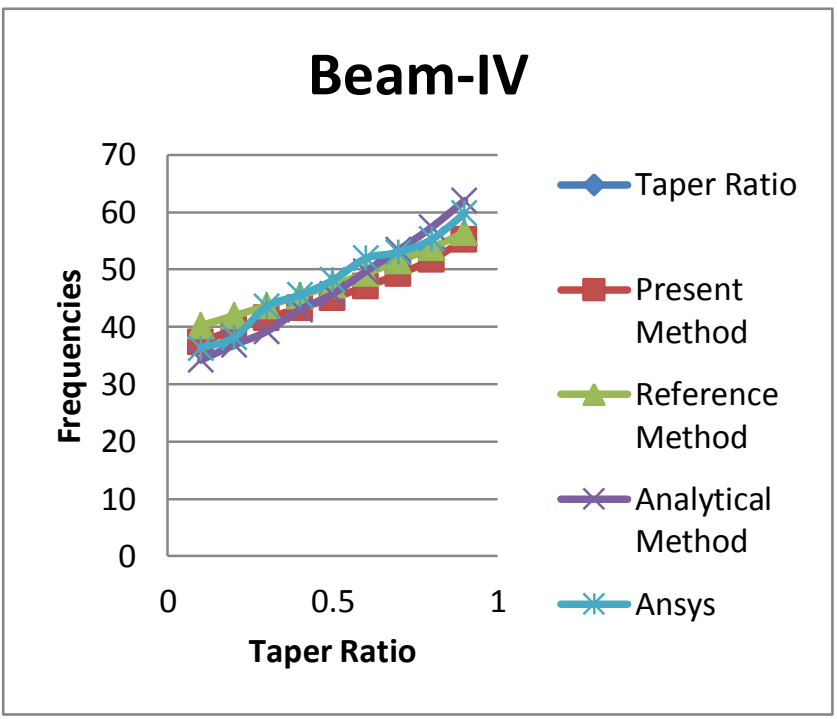

\section{Simulation results}

The concept of Energy field method is used to determine the fundamental frequencies of tapered shear flexible beams, with most practically used boundary condition. The boundary conditions of the beams considered is Cantilever beam, one with axially immovable ends. For more accuracy of the results these values are checked by using ANSYS 10 software. The procedure of the simulation is explained below.

1. Open the ANSYS workbench tool.

2. Click on structural analysis - one window will be appear.

3. Give engineering data related your applied material.

4. Click on Geometry - import (or) create the geometry - browse.

5. Click on model - Mesh - Generate mesh - give mesh size update mesh.

6. Click on model - supports - select fixed support - click on one face of the geometry.

7. Click on model - Analysis settings - give max number of modes - solve.

8. Go to solutions - select total deformation.

We can note the frequencies values which will be appear on the screen.

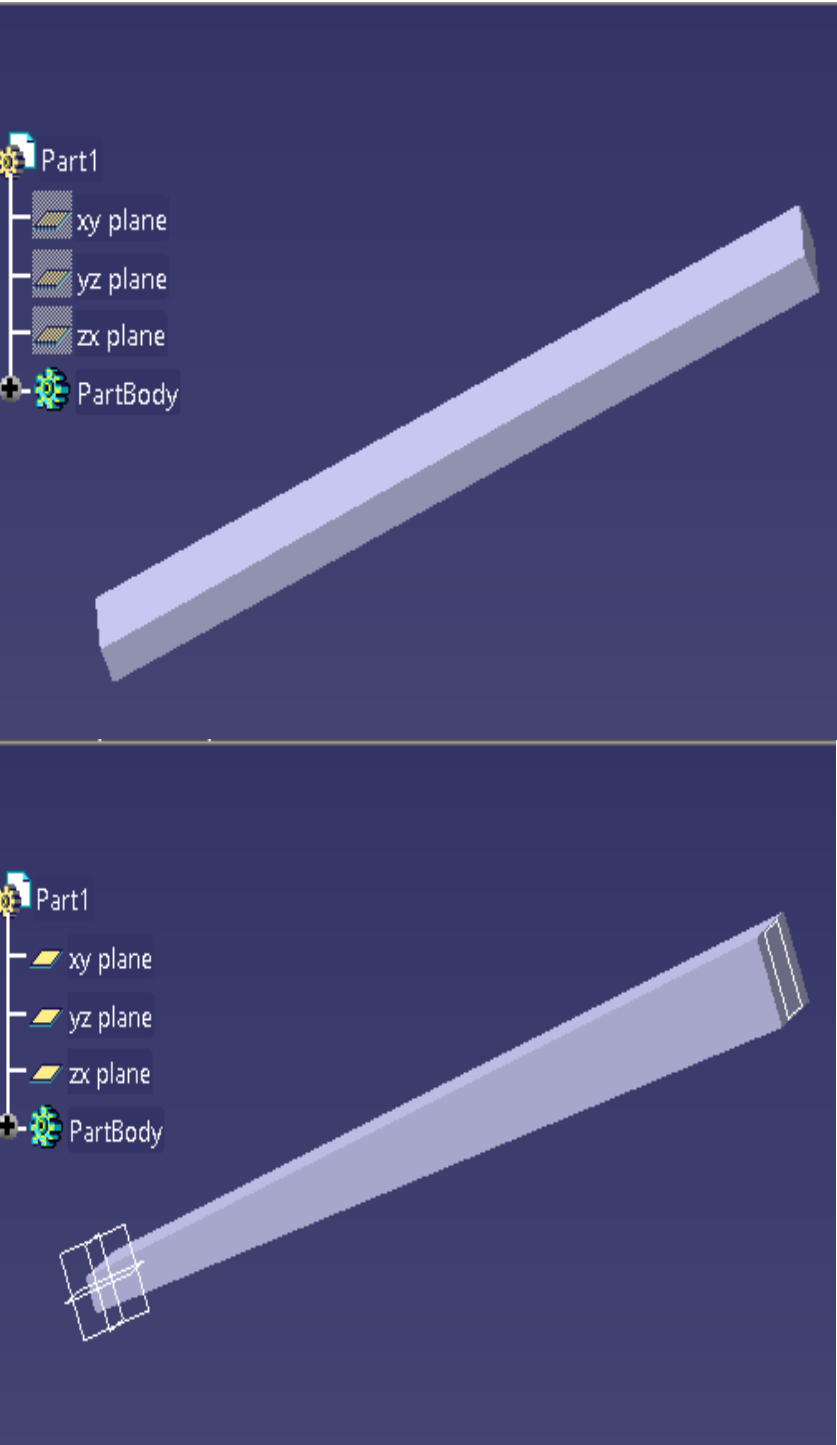

Fig.3: Taper beam models designed in CATIA V5 R20

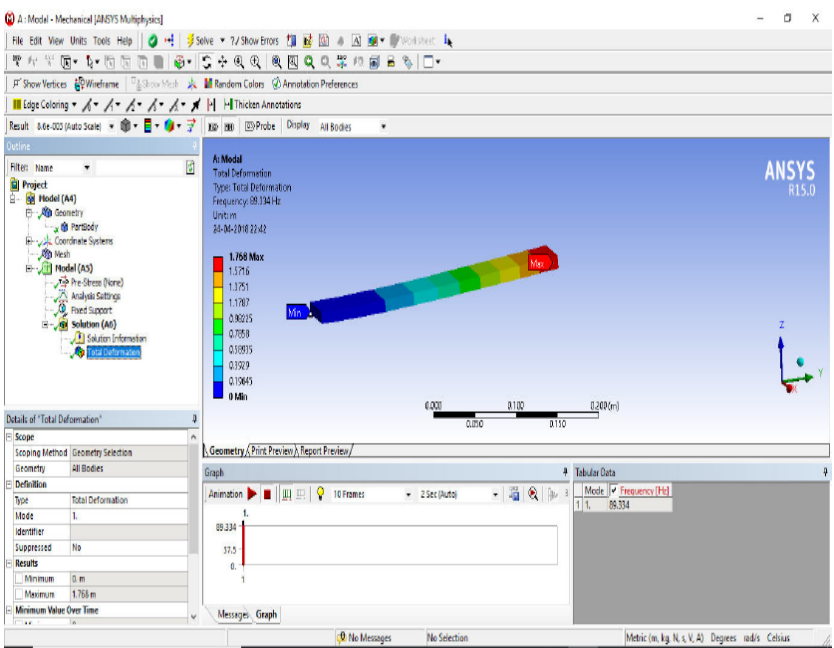

\#0

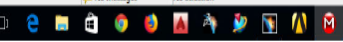




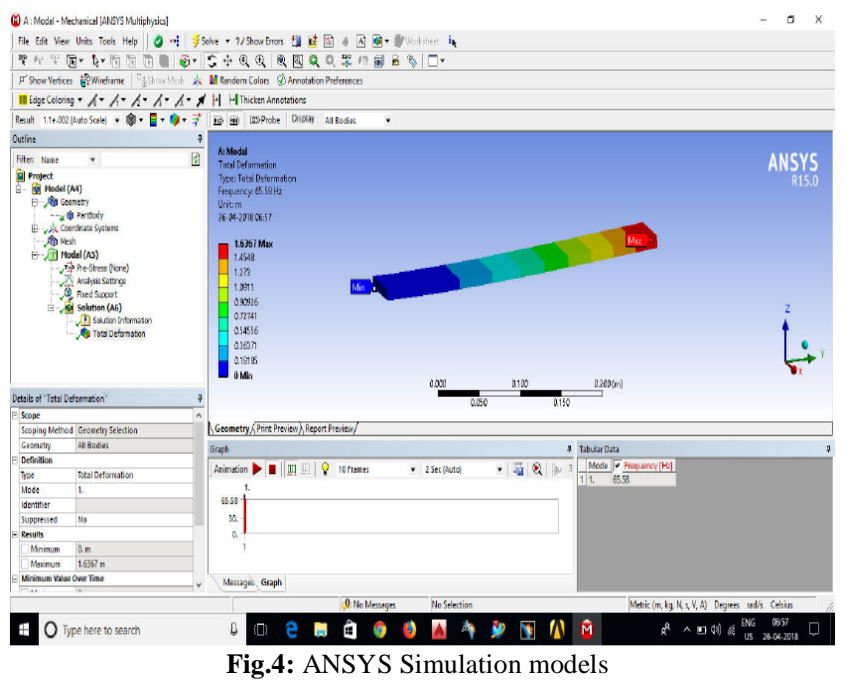

\section{Conclusion and future scope}

This method is developed for linear free vibration analysis of tapered Timoshenko cantilever beams. Moreover the tapered functions of breadth and height of the cross section of the beam have been taken into account for deriving the natural frequency function with cantilever beam boundary conditions. These values are compared with existing method called coupled displacement field method and Rayleigh-Ritz method for The convergency, accuracy, efficiency, and versatility of the method these frequencies values are also compared with Ansys software. The accuracy of the present method results is very rapid. This present research work can be extend for hinged-hinged beam and simply supported beams.

\section{References}

[1] Bulent Yardimoglu "vibration analysis of rotating tapered Timoshenko beams by a new finite element model", in IOS press shock and vibration 13 (2006) 117-126.

[2] K. Rajesh, K. Meera sahib "linear free vibration analysis of tapered Timoshenko beam using coupled displacement field method ISSN online 2424-4627.

[3] P. Ravi Kumar, K. Mohana Rao, N. Mohan Rao "Free vibration analysis of functionally graded rotating beam by Differential transform method" IJEMS Vol 24 Pp 107-114.

[4] Mani Kant "Study and Vibration analysis of Gradually decreasing beam by FEM" IJEMRT volume 9 (2018) Pp. 431-444.

[5] Ming-Hung Hsu "Vibration analysis of non-uniform beams resting on elastic foundations using the spline collocation method" Tamkang journal of science and engineering, Vol. 12, No. 2, pp. 113-122.

[6] Dawid Cekus "Free vibration of a Cantilever stepped Timoshenko beam" Scientific research of the institute of Mathematics and computer sciences 4(11) 2012, 11-17.

[7] U. Jyothirmayi, P. Ravikumar "Vibration analysis of rotating tapered composite beam” IRJET Vol. 03, IS. 08, P-ISSN 2395-0072. 